\title{
In vivo assessment of a novel biodegradable ureteral stent
}

\author{
Alexandre A. Barros ${ }^{1,2}$ • Carlos Oliveira ${ }^{2,3} \cdot$ Ana J. Ribeiro $^{4} \cdot$ Riccardo Autorino $^{5}$. \\ Rui L. Reis ${ }^{1,2}$ Ana Rita C. Duarte ${ }^{1,2}$ Estevão Lima ${ }^{2,3}$
}

Received: 1 August 2017 / Accepted: 2 November 2017

(c) Springer-Verlag GmbH Germany, part of Springer Nature 2017

\begin{abstract}
Purpose To perform an in vivo assessment of a newly developed biodegradable ureteral stent (BUS) produced with natural-based polymers.

Methods The BUS is based on a patented technology combining the injection process with the use of supercritical fluid technology. Study was conducted at ICVS-University of Minho (Braga, Portugal) and a total of ten domestic pigs were used. In seven animals, the experimental BUS stent was inserted, whereas in the remaining a commercially available stent was used (6-Fr Biosoft ${ }^{\circledR}$ duo stents, Porges Coloplast, Denmark). Post-stenting intravenous pyelogram was used to evaluate the degree of hydronephrosis. The in vivo stent degradation was measured as function of the weight loss. Moreover, the tensile properties of the BUS were tested during in vivo degradation. After maximum 10 days, animals were killed and necropsy was performed. Tissues were compared between the stented groups as well as between the non-stented contralateral ureters and stented
\end{abstract}

Ana Rita C. Duarte

aduarte@dep.uminho.pt

1 3B’s Research Group-Biomaterials, Biodegradables and Biomimetics, University of Minho, Headquarters of the European Institute of Excellence on Tissue Engineering and Regenerative Medicine, AvePark, Barco, 4805-017 Guimarães, Portugal

2 ICVS/3B's-T Government Associate Laboratory, Braga/Guimarães, Portugal

3 School of Health Sciences, Life and Health Sciences Research Institute (ICVS), University of Minho, Braga, Portugal

4 University School of Vasco da Gama, Coimbra, Portugal

5 Division of Urology, Virginia Commonwealth University, Richmond, VA, USA ureters in each group. Biocompatibility was assessed by histopathological grading.

Results In all cases, the BUS was only visible during the first $24 \mathrm{~h}$ on X-ray, and in all cases the BUS was completely degraded in urine after 10 days, as confirmed on necropsy. During the degradation process, the mechanical properties of the BUS decreased, while the commercial ureteral stents remained constant. At all time-points after stent insertion, the level of hydronephrosis was minimal. Overall, animals stented with BUS had an average grade of hydronephrosis which was lower compared to the controls. The BUS showed better pathological conditions, and hence better biocompatibility when compared with commercial stents.

Conclusions Notwithstanding the limitations of the present study, the in vivo testing of our novel natural origin polymer-based BUS suggests this device to feature homogeneous degradation, good urine drainage, and high biocompatibility. Next steps will be to increase its stability, and to improve the radiopacity without compromising its degradation. Ultimately, clinical studies will be required to determine the safety and feasibility of its use in humans.

Keywords Biodegradable - Polymers - Ureteral stent . Ureteroscopy

\section{Introduction}

Ureteral stents are routinely used in daily urological practice for a variety of indications. Nevertheless, it is widely recognized that these medical devices can produce significant symptoms, like infection, encrustation, patient discomfort [1]. Moreover, a cystoscopy procedure is usually required for stent removal. Eventually, the so-called "forgotten stent syndrome" can lead to kidney loss or even death [2]. Because 
of these issues, much research over the past two decades has been focused on "biodegradable" materials to minimize stent-related morbidity, and to avoid a second procedure for stent removal [3].

Several biodegradable ureteral stents (BUS) have been reported, but all of them have failed because of poor biocompatibility or incomplete degradation $[4,5]$. The main challenge remains to have a uniform and homogenous degradation, preventing the formation of fragments that can cause obstruction. We recently described a BUS produced with natural-based polymers [6].

In the present study, we assessed in vivo (in a porcine model) this newly developed BUS, by comparing its degradation, mechanical properties, drainage, physiological and histological features with those of a commercially available ureteral stent.

\section{Methods}

\section{BUS technology}

The BUS described in this study is based on a patented technology combining the injection process with the use of supercritical fluid technology. Briefly, an aqueous solution of gelatin-alginic-acid sodium salt and bismuth carbonate basic (65:30:5 wt \%) —is prepared. After obtaining a homogenous solution, $15 \mathrm{mM}$ of a crosslinking agent (genipin) is added. The solution is then injected in a mold to obtain a 6-Fr ureteral stent with the total length of $20 \mathrm{~cm}$, wall thickness of $500 \mu \mathrm{m}$ and internal diameter of $1000 \mu \mathrm{m}$. Supercritical technology is the used to dry the stent. Supercritical technology is emerging as an alternative to conventional materials' processing methods for the preparation of threedimensional structures and injectable particles suitable to be used in regenerative medicine [7]. The coating is performed by immersion into a $10 \mathrm{wt} \%$ solution of polycaprolactone resin 787 with $2 \mathrm{wt} \%$ of bismuth carbonate basic in chloroform. Last, stent is sterilized with ethylene oxide at $42{ }^{\circ} \mathrm{C}$ for $3 \mathrm{~h}$.

\section{Experimental protocol}

The study was conducted at ICVS-University of Minho (Braga, Portugal) in accordance with its internal ethical protocol for animal experiments. A total of ten domestic pigs were used. All procedures were performed under general anesthesia and mechanical ventilation. After emptying the bladder, a semi rigid 7-Fr ureteroscope (Karl Storz, Tuttlingen, Germany) was inserted through the urethra. A $0.035^{\prime \prime}$ flexible tip guidewire (AQUATRACK ${ }^{\circledR}$ Hydrophilic Nitinol, Cordis ${ }^{\circledR}$, Johnson \& Johnson) was inserted, and then stent was passed over the guide wire into the kidney. In seven animals, the experimental BUS stent was inserted, whereas in the remaining a commercially available stent was used (6-Fr Biosoft ${ }^{\circledR}$ duo stents, Porges Coloplast, Denmark). Correct positioning of stents was confirmed by X-ray. Post-stenting intravenous pyelogram (IVP) was used to evaluate the degree of hydronephrosis at days $0,1,5$ and 10 [5].

After maximum 10 days, animals were killed and necropsy was performed. Among the seven pigs stented with the BUS, one died for pre-existing pneumonia at day 5, and one was killed at day 7. In both cases, the BUS was retrieved and used for analysis. A midline incision was carried out to excise the kidneys, ureters and bladder en bloc. The kidneys and ureters were measured and a representative section of each organ was fixed in 10\% formalin and stained with hematoxylin-eosin and Masson's trichrome. Tissues were compared between the stented groups (biodegradable vs commercial stents) as well as between the non-stented contralateral (control) ureters and stented ureters in each group. Biocompatibility was assessed by histopathological grading, as suggested by Chew et al. [8] (from Grade 0-no inflammation to Grade 3 -severe edema, congestion, inflammation).

The in vivo stent degradation was measured as function of the weight loss. Stents were first measured at baseline in "dry" condition. When removed from the in vivo model, it was dried and then measured. The weight loss of stents was calculated according to the following equation:

$\%$ Weight loss $=\frac{W_{\mathrm{f}}-W_{\mathrm{i}}}{W_{\mathrm{i}}} \times 100$,

where $\mathrm{W}_{f}$ is the final weight of the stent (dried after immersion/placement) and $W_{i}$ is the initial weight of the stent.

The tensile properties of the BUS were tested at days 0,5 and 7 . The testing at days 5 and 7 was performed on the stents retrieved from the one pig died for pneumonia at day 5 , and the one killed at day 7 . Tensile mechanical analysis was performed by an INSTRON 5540 (Instron Int. Ltd, High Wycombe, UK) universal testing machine with a load cell of $1 \mathrm{kN}$. The load was placed midway between the supports with a span (L) of $30 \mathrm{~mm}$. The crosshead speed was $1: 5 \mathrm{~mm} \mathrm{~min}^{-1}$. For each condition, the specimens were loaded until core break. The in vivo recovered stents were cut into equal parts along their length and compared. The tensile properties of BUS were compared with the control group used in this study from the data of our previous study [9].

All quantitative data are presented as mean \pm standard deviation (SD). Statistical analysis was performed using Graph Pad Prism 6.00 software (San Diego, USA). Statistical significances $(* p \leq 0.05, * * p \leq 0.01$ and $* * * p \leq 0.001)$ were determined using one-way analysis 
of variance (ANOVA) for an average of three to twelve replicates, followed by post hoc Tukey's test for all pairwise mean comparisons. Hydronephrosis scores with time were compared using two-way repeated measures ANOVA followed by the Bonferroni post test.

\section{Results}

In all cases, the BUS was only visible during the first $24 \mathrm{~h}$ on X-ray, and in all cases the BUS was completely degraded (100\% weight loss) in urine after 10 days, as confirmed on necropsy. In the pig killed early at day 7, the mass reduction of the BUS was $38 \%$ (Fig. 1). Homogeneous degradation by erosion along the entire stent surface was visualized, while the lumen remained patent. During the degradation process, the mechanical properties of the BUS decreased, while the commercial ureteral stents remained constant. BUS demonstrated to have lower maximum tensile strain and higher stiffness compared to the commercial stent (Fig. 2). At all time-points after stent insertion, the level of hydronephrosis-as based on the IVP assessment - was grade 2 or lower. Overall, animals stented with BUS had an average grade of hydronephrosis which was lower compared to the controls
Fig. 1 a all of the five BUS were completely degraded (100\% weight loss) in urine after 10 days; $\mathbf{b}$ the BUS retrieved after 5 days was intact with a weight loss of $24 \%$; c the BUS retrieved at day 7 was also intact with a mass reduced by $38 \%$
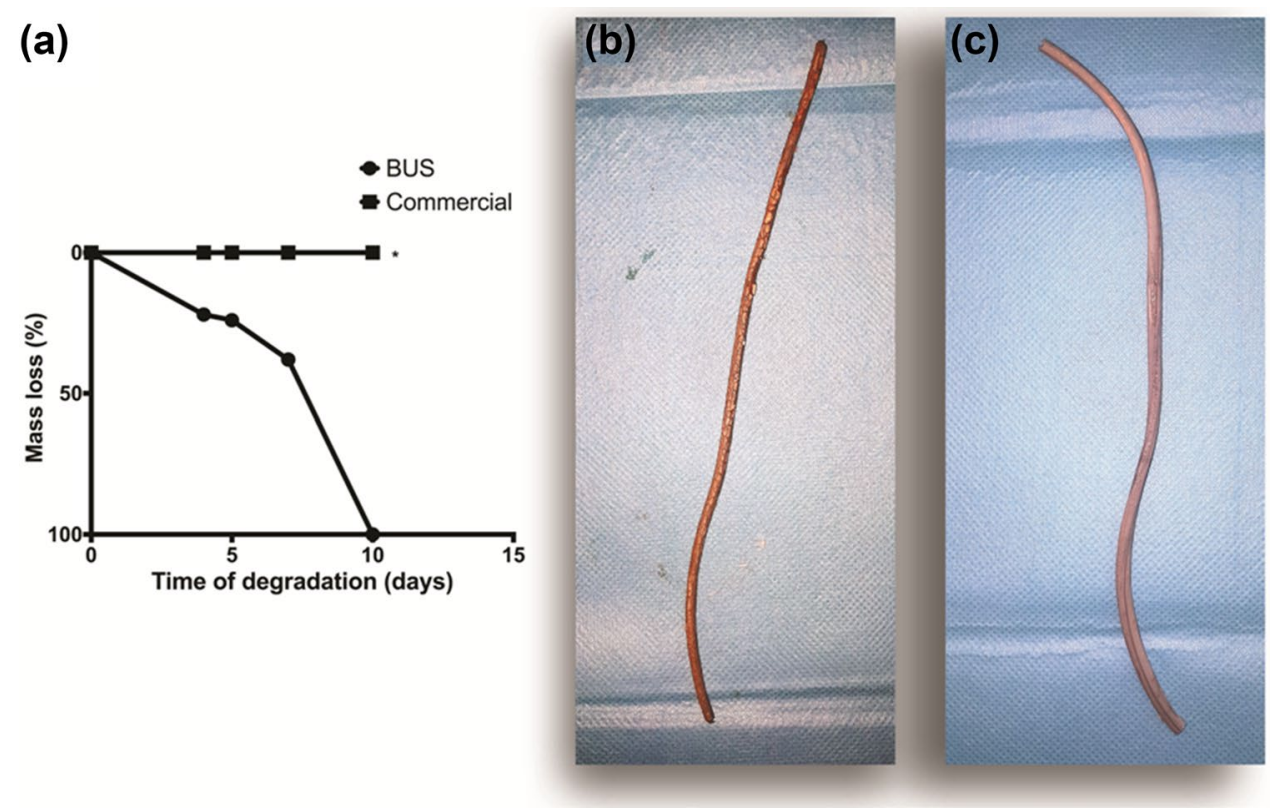

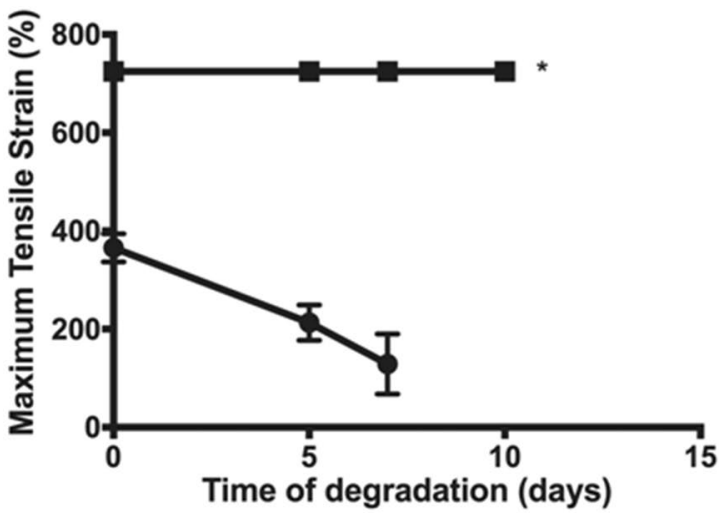

Fig. 2 The in vivo tensile mechanical properties, namely maximum tensile strain (\%) and also the stiffness-the Young's modulus (MPa) — of the BUS during the degradation process are presented here. BUS showed less $40 \%$, at day 5 and $65 \%$, at day 7 of maximum tensile strain after in vivo degradation. In terms stiffness, BUS were significantly more resistant than the control until day 7 , the time that

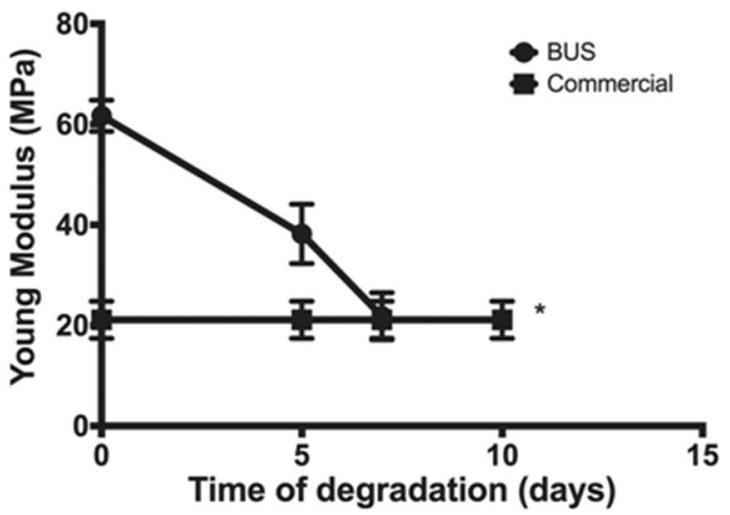

both stents presented similar values. During the degradation process, the mechanical properties of the BUS decreased, while the commercial ureteral stents remained constant. BUS demonstrated to have lower maximum tensile strain and higher stiffness compared to the commercial stent 

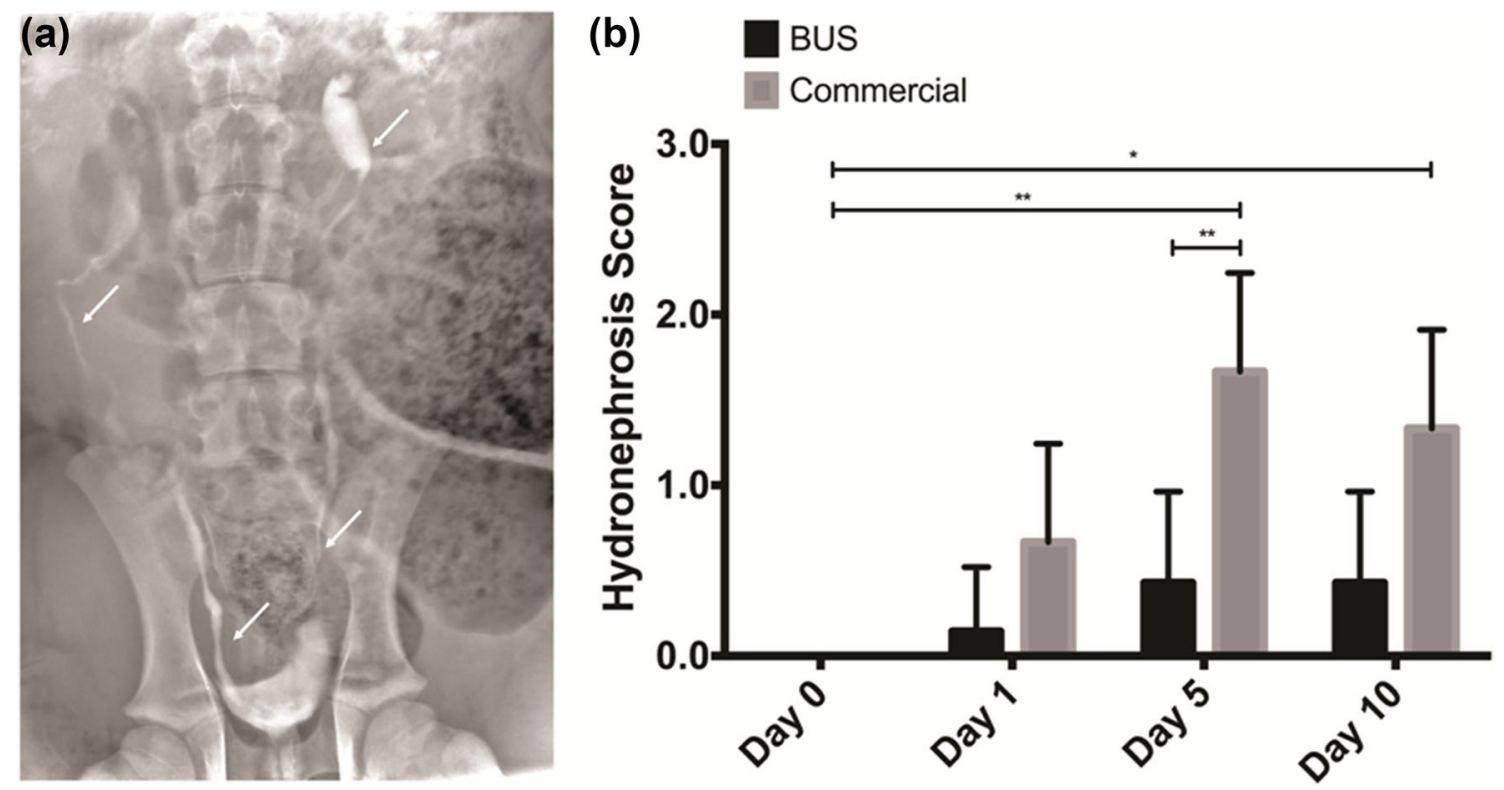

Fig. 3 a representative image of pyelogram at day 10 after BUS degradation; b hydronephrosis score obtained for all animals stented with BUS and commercial stents. At all time-points after stent inser-

Table 1 Biocompatibility parameters of BUS and commercial stents group in the kidney and the ureters

\begin{tabular}{llll}
\hline Group & BUS & Commercial & $p$ value \\
\hline Nephropathy & $0.17 \pm 0.37$ & $0.67 \pm 0.47$ & 0.24 \\
Ureteral pathology & $0.33 \pm 0.47$ & $1.67 \pm 0.33$ & $0.04^{*}$ \\
\hline
\end{tabular}

Data presented as mean $\pm \mathrm{SD}$

*Significant

(Fig. 3). Based on histopathological grades for nephropathy and ureteral pathology, kidneys and ureters stented with BUS showed better pathological conditions, and hence better biocompatibility when compared with commercial stents (Table 1). No significant differences were observed between the stented kidney and non-stented and between the two types of stents in terms of ureteral width (Fig. 4). Not surprisingly, histological analysis revealed greater changes in stented ureters than in non-stented ureters in each group. Overall, no significant inflammatory or necrotic cells were found in both groups (Fig. 5).

\section{Discussion}

Fragments from stent degradation still represent an issue that needs to be overcome, and this is the main reason why biodegradable stents are not available in the market yet. Fragments can act as a nucleation point for bacterial adhesion and/or encrustation development leading to further tion, the hydronephrosis level of all pigs was not higher than grade 2. Overall, animals stented with BUS had an average of slightly less hydronephrosis compared with the control, commercial stent

complications [10]. Chew et al. developed a biodegradable stent-Uriprene ${ }^{\mathrm{TM}}$ - - that was investigated in vivo by assessing degradation time, and physiological and histological responses in a porcine model [5]. Zhang et al. developed a similar glycolic-lactic acid fiber based BUS, and reported results in vivo, in a canine model [11]. Both studies presented good results regarding the degradation and biocompatibility; however, upon degradation they reported fragments of the stent in the renal pelvis and bladder. The time of degradation of these glycolic-lactic acids-based BUSs was around 4 weeks. Hjdinjak et al. designed an experiment to simulate the physical forces that may lead to material fatigue of the stent. From their results, it appeared that stent fractures can be avoided by observing a maximum indwelling time of 6-8 weeks [12]. In clinical practice, temporary ureteral stents are generally kept up to 4 weeks after their insertion, depending on the indication. In our study, the time of stent degradation was shorter (10 days), which can limit the therapeutic applications of this device now. However, modification of the stent formulation, incorporating new polymers or different cross-linking, may result in longer indwelling times. Overall, animals stented with BUS present less degree of hydronephrosis compared with the controls. It is well documented that placement of conventional ureteral stents may result in a certain level of hydronephrosis [13]. While ureteral stents can alleviate obstruction, the urine flow is not the same as freely as that in a non-stented ureter [14]. The degradation process of BUS showed no effect on the urine flow, and it did not increase the hydronephrosis. Interestingly, IVP findings suggested that BUS might provide 
Stented

Non-Stented

Kidneys

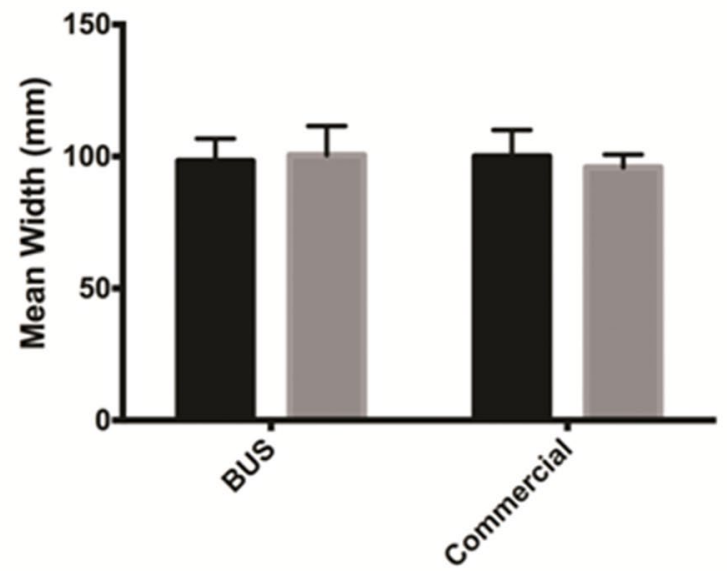

Ureters - mid

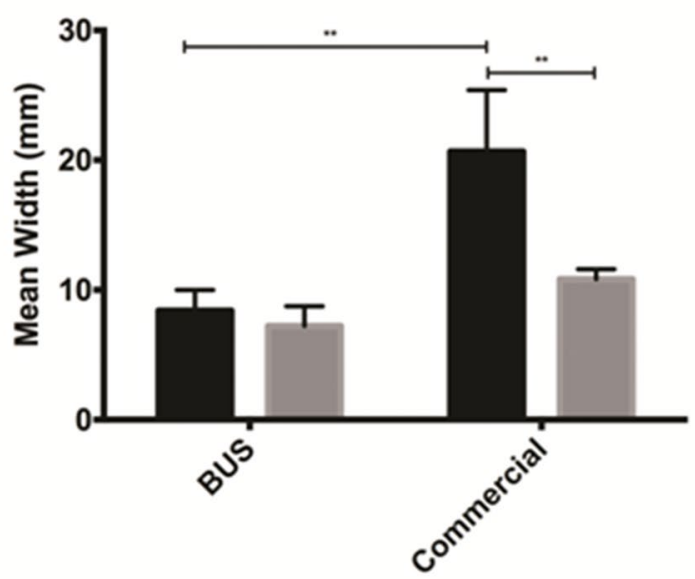

Fig. 4 Overall the ureteral width of BUS-stented ureters was not statistically significantly larger when compared to the non-stented ureter. In contrast, commercial stented ureters were statistically significantly

better flow than control stent. This can be explained by the water uptake capacity of hydrogel-based materials which has higher absorbing properties then the conventional material used in the non-BUSs [15].

Ureteral stents are in close contact with uroepithelium and because of, therefore, their biocompatibility is an important feature [11]. Based on histopathological grades for nephropathy and ureteral pathology, kidneys and ureters stented with BUS shown better pathological conditions, and hence better biocompatibility. Overall, the ureteral width of BUS-stented ureters was not statistically significantly larger when compared to the non-stented ureter. Similar results were observed by Chew et al. demonstrating the ability of BUSs to be less abrading when compared with conventional ureteral stents [5]. It is well documented that the

\section{Ureters - Upper}

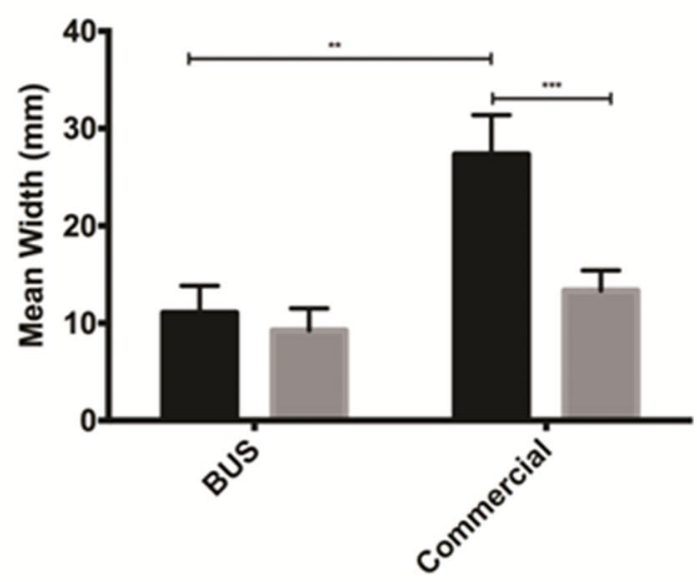

Ureters - distal

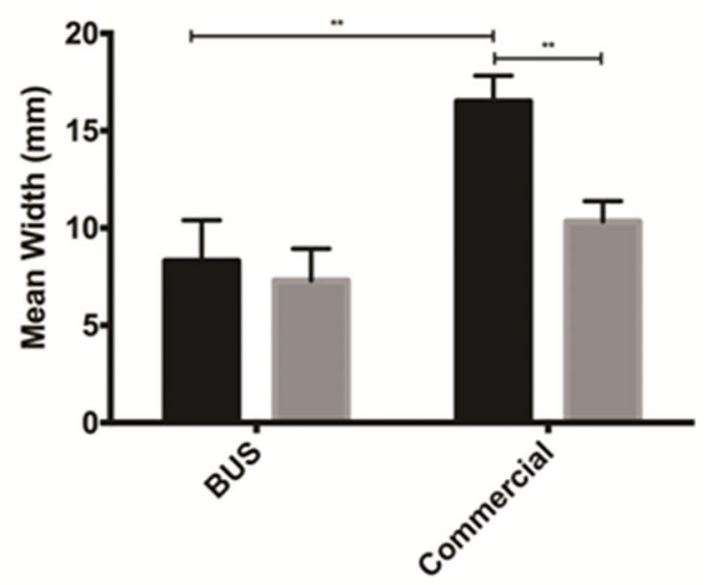

greater when compared to the ureters non-stented, in mid ( $p \leq 0.01)$, upper and distal $(p \leq 0.001)$ ureter sections

placement of commercial ureteral stents can cause irritation of the ureteral epithelium [16]. The ureteral epithelium after BUS placement and degradation remained intact without any major change compared to the control. The thickness of ureter mucosa remained similar and no inflammation or hydronephrosis was observed. In contrast, commercial stent showed to cause some degree of edema and epithelium destruction, as also observed by others $[5,16]$. Another study on biodegradable stents developed by using different materials showed severe inflammatory reaction, with stent material entrenched in the ureteral wall [4]. This reaction was not observed in our study. The characteristics of the base material of the stents are essential for a good preservation of ureteral histopathology. Our BUS is a hydrogel-based stent, shown to be ureteral urothelium-friendly material, and 


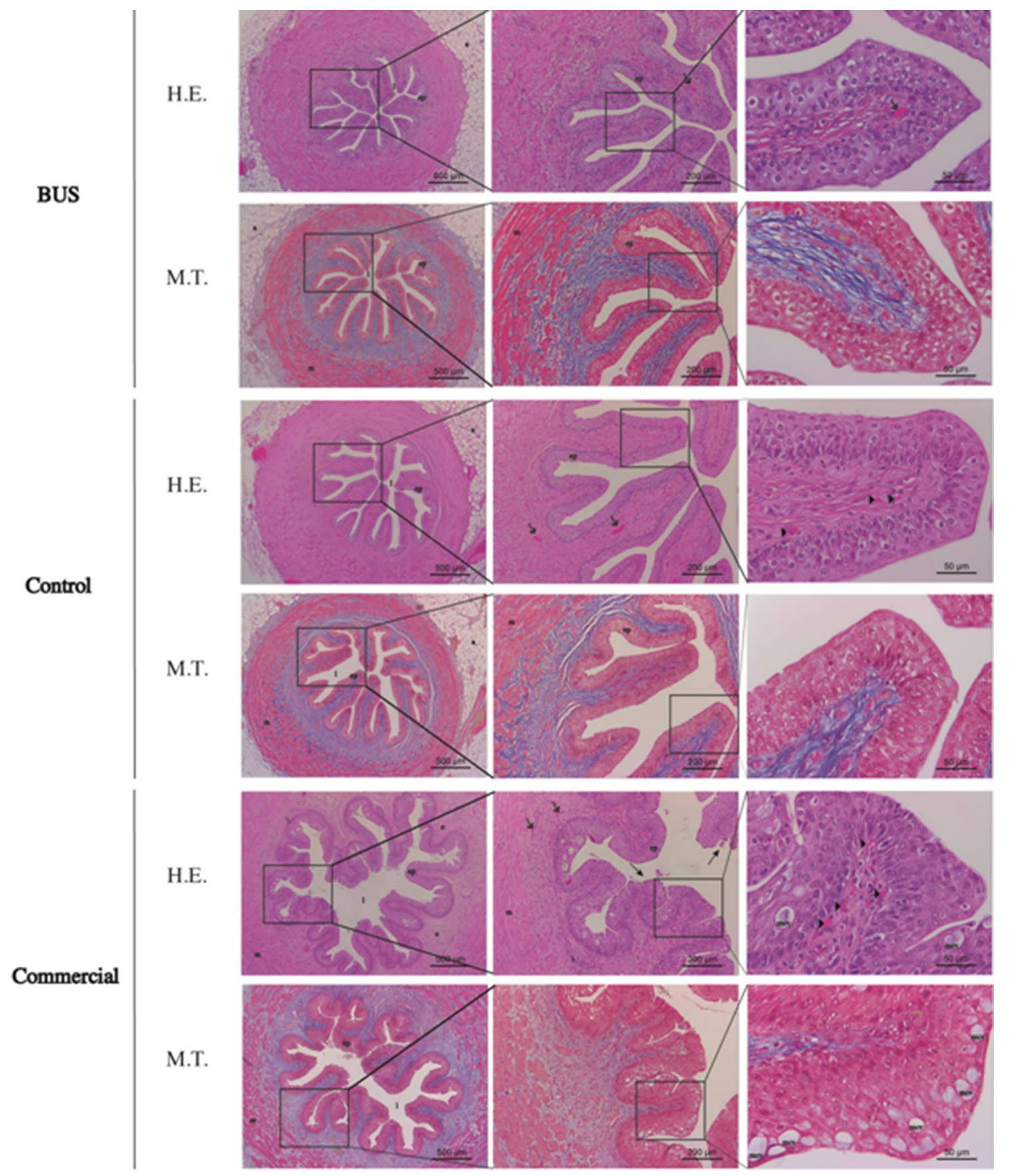

Fig. 5 Representative HE (Hematossilin Eosin) and MT (Massons trichrome stain) images of the ureters. It is possible to appreciate the differences in the mucosa thickness between the control and the commercial ureteral stent group

the degradation products not induced any ureteral inflammatory response. Hydrogel-based materials have been used for conventional stents to reduce the stent-related problems; however, full hydrogel stents have not yet been introduced in the market yet [3].

Our study has some limitations, including the small sample, and the use of only one type of commercial stent as a control. Next steps will be to increase the stability of the BUS in vivo, improve the radiopaque features while the degradation by erosion is not compromised, and it occurs without fragments of the stent. Moreover, modification of the stent formulation, incorporating new polymers or different cross-linking, should ideally result in longer (more than 10 days) indwelling times, allowing for a broader clinical application of the product. Ultimately, 
clinical studies will be required to determine the safety and feasibility of its use in humans.

\section{Conclusions}

Herein, we describe the development and in vivo testing of a novel natural origin polymer-based BUS. This device presents a homogeneous degradation by surface erosion, good physiological and histopathological response, effective urine drainage, and high biocompatibility. Overall, this novel BUS can be regarded as a significant advance in the field. Nevertheless, further studies are needed to address current limitations of this product and to prove its safety in the clinical setting.

Acknowledgements The research leading to these results has received funding from the ICVS/3B's Associated Laboratory with the project reference POCI-01-0145-FEDER-007038, from the project "Novel smart and biomimetic materials for innovative regenerative medicine approaches" RL1-ABMR-NORTE-01-0124FEDER-000016) cofinanced by North Portugal Regional Operational Programme (ON.2-O Novo Norte), under the National Strategic Reference Framework (NSRF), through the European Regional Development Fund (ERDF). Alexandre Barros acknowledges his FCT PhD grant SFRH/BD/97203/2013. The authors acknowledge Teresa Oliveira and Tírcia C. Santos for the technical assistance on histopathology analysis.

\section{References}

1. Damiano R, Oliva A, Esposito C et al (2002) Early and late complications of double pigtail ureteral stent. Urol Int 69:136-140

2. Singh V, Srinivastava A, Kapoor R et al (2005) Can the complicated forgotten indwelling ureteric stents be lethal? Int Urol Nephrol 37:541-546

3. Chew BH, Lange D (2016) Advances in ureteral stent development. Curr Opin Urol 26:277-282
4. Olweny EO, Landman J, Andreoni C et al (2002) Evaluation of the use of a biodegradable ureteral stent after retrograde endopyelotomy in a porcine model. J Urol 167:2198-2202

5. Chew BH, Paterson RF, Clinkscales KW et al (2013) In vivo evaluation of the third generation biodegradable stent: a novel approach to avoiding the forgotten stent syndrome. J Urol 189:719-725

6. Barros AA, Rita A, Duarte C et al (2015) Bioresorbable ureteral stents from natural origin polymers. J Biomed Mater Res B Appl Biomater 103(3):608-617

7. Kankala RK, Zhang YS, Wang SB, Lee CH, Chen AZ (2017) Supercritical fluid technology: an emphasis on drug delivery and related biomedical applications. Adv Healthc Mater. https://doi. org/10.1002/adhm.201700433

8. Chew BH, Knudsen BE, Nott L et al (2007) Pilot study of ureteral movement in stented patients: first step in understanding dynamic ureteral anatomy to improve stent comfort. J Endourol 21:1069-1075

9. Barros AA, Oliveira C, Lima E et al (2016) Gelatin-based biodegradable ureteral stents with enhanced mechanical properties. Appl Mater Today 5:9-18

10. Schlick RW, Planz K (1997) Potentially useful materials for biodegradable ureteric stents. Br J Urol 1997(80):908-910

11. Zhang MQ, Zou T, Huang YC et al (2014) Braided thin-walled biodegradable ureteral stent: preliminary evaluation in a canine model. Int J Urol 21:401-407

12. Hajdinjak T, Patel M, Papatsoris A, Masood J, Buchholz N, Birch M (2008) In vitro simulation of stent fracture mechanisms in ureteric nitinol wire stents. Urol Res 36(5):241-245

13. Richter $\mathrm{S}$, Ringel A, Shalev $M$ et al (2000) The indwelling ureteric stent: a 'friendly' procedure with unfriendly high morbidity. BJU Int 85:408-411

14. Ramsay JWA, Payne SR, Gosling PT et al (1985) The Effects of Double-J Stenting on Unobstructed Ureters - an Experimental and Clinical-Study. Br J Urol 57:630-634

15. Young S, Wong M, Tabata Y, Mikos AG (2005) Gelatin as a delivery vehicle for the controlled release of bioactive molecules. $\mathrm{J}$ Control Release 5 109(1-3):256-274

16. Elwood CN, Lange D, Nadeau R et al (2010) Novel in vitro model for studying ureteric stent-induced cell injury. BJU Int 105:1318-1323 